Revue d'histoire de l'Amérique française

REVUE D.HISTOIRE DE L'AMÉRIQUE FRANÇAISE

\title{
Artilleurs canadiens-français dans la bataille d'Allemagne (novembre 1944 - mai 1945) (suite)
}

\section{Jacques Gouin}

Volume 18, numéro 1, juin 1964

URI : https://id.erudit.org/iderudit/302343ar

DOI : https://doi.org/10.7202/302343ar

Aller au sommaire du numéro

Éditeur(s)

Institut d'histoire de l'Amérique française

ISSN

0035-2357 (imprimé)

1492-1383 (numérique)

Découvrir la revue

Citer cet article

Gouin, J. (1964). Artilleurs canadiens-français dans la bataille d'Allemagne (novembre 1944 - mai 1945) (suite). Revue d'histoire de l'Amérique française, 18(1), 85-111. https://doi.org/10.7202/302343ar d'utilisation que vous pouvez consulter en ligne.

https://apropos.erudit.org/fr/usagers/politique-dutilisation/ 


\section{ARTILLEURS CANADIENS-FRANÇAIS DANS LA BATAILLE D'ALLEMAGNE (novembre 1944 - mai 1945) (suite)*}

$8 e$ article

\section{BATAILLE DE LA RHÉNANIE}

\section{BUT DE L'OPÉRATION "VERITABLE"}

L'opération "Veritable", déclenchée le 8 février 1945 sur le front anglo-canadien, fut le prélude à l'écrasement définitif de l'Allemagne nazie. C'est en effet dans le nord-ouest de l'Europe, - plus précisément dans le secteur occupé par le $21^{\mathrm{e}}$ groupe d'armées du maréchal Montgomery, - que s'ouvrit le dernier acte du drame hitlérien. Et c'est le dégagement du port d'Anvers, par les troupes canadiennes, qui avait permis au général Crerar d'accumuler le matériel nécessaire à cette opération ${ }^{1}$. Le général Eisenhower, pour sa part, devait plus tard écrire dans son rapport officiel:

Conformément à nos plans stratégiques relatifs aux opérations à l'est du Rhin, l'effort principal des Alliés à l'ouest du fleuve devait se produire dans le secteur nord, en vue d'établir des têtes de pont au nord de la Rhur ${ }^{2}$.

Les plans stratégiques qui visaient à mettre fin à la guerre en Europe comportaient trois phases: 1. opération "Veritable", par la $1^{\text {re }}$ armée canadienne, suivie de l'opération "Grenade", par la $9^{\mathrm{e}}$ armée américaine, celle-ci devant ensuite progresser vers le Rhin jusqu'à Düsseldorf; 2 . ce groupe d'armées du Nord devait ensuite refouler l'ennemi jusqu'au Rhin, de concert avec

*Voir notre Revue, XVI: 240-253, 353-368, 536-548; XVII: 70-86, 244-257, 325-339, 504-530.

1 R. W. Thompson, The Battle for the Rhineland, (Londres, Hutchinson, 1958), 144.

2 Général Eisenhower, Les Opérations en Europe des Forces expéditionnaires alliées ( 6 juin 1944 - 8 mai 1945), traduit par le capitaine Ramsay, Paris, Charles-Lavauzelle et Cie, (1948), 208. 
une avance des armées du Sud; et enfin 3. les armées du Sud devaient se rendre à leur tour jusqu'au bord du Rhin. Bref, les plans stratégiques du général Eisenhower visaient à détruire l'armée allemande à l'ouest du Rhin, sans tenir compte d'aucune considération d'ordre politique ${ }^{3}$.

\section{RÔLE DE L'ARMÉE CANADIENNE}

Dans cette offensive sur le Rhin, la $1^{\text {re }}$ armée canadienne se trouvait de nouveau sur un champ de bataille "difficile et désagréable ${ }^{4}$ ". L’objectif immédiat du général Crerar était la ligne Xanten-Geldern. Immédiatement au-delà de la frontière allemande faisant face à l'armée canadienne, se trouvait la forêt du Reichswald, de 8 milles de longueur sur 4 de profondeur, et, douze milles plus à l'est, les abords de Xanten étaient bloqués par deux autres forêts, celles du Hochwald et du Balberger, formant une autre zone boisée de 6 milles de longueur sur 3 de profondeur. Ces forêts étaient alors plantées de jeunes pins espacés de 4 à 7 pieds ${ }^{5}$.

En plus de ces obstacles naturels, les troupes canadiennes allaient se heurter aux formidables fortifications permanentes de la ligne Siegfried, comprenant une double série de tranchées, protégées par un fossé antichars creusé devant la forêt du Reichswald ${ }^{6}$.

Au début de février 1945, le secteur de la forêt du Reichswald était défendu par la $84^{\mathrm{e}}$ division du major-général Heinz Fiebig, laquelle formait l'aile droite $d u 6^{\text {e }}$ corps commandé par Erich Straube. Cet ensemble de troupes formait la $1^{\text {re }}$ armée de parachutistes du général Schlemm. L'aile gauche de Straube était constituée par la $180^{\mathrm{e}}$ division, déployée le long de la Meuse. Et, sur l'autre rive du Rhin, se trouvaient le $83^{\mathrm{e}}$ corps de la $25^{\mathrm{e}}$

3 Ibid., et Thompson, op. cit., 82.

${ }^{4}$ Colonel C. P. Stacey, Histoire officielle de la participation de l'armée canadienne à la seconde guerre mondiale: la campagne de la victoire: les opérations dans le nord-ouest de l'Europe (1944-1945) (Ottawa, Imprimeur de la Reine et contrôleur de la papeterie, 1960), 487.

5 Ibid., 488.

6 Ibid., 489. 
armée, et la $2^{\text {e }}$ division de parachutistes, voisine de celle de Fiebig ${ }^{7}$.

\section{LE RÉGIMENT SE PRÉPARE À ENTRER EN ALLEMAGNE}

Dans un ordre du jour du 6 février, signé du capitaine Laplante, tous les membres du Régiment étaient mis au courant de ce qui allait bientôt se passer. On pouvait lire notamment ce qui suit:

Nous allons maintenant pénétrer en territoire ennemi. Tous les civils, y compris les réfugiés et les femmes, sont nos ennemis. Donc, PAS de fraternisation...

Le succès de cette opération pourrait avoir un effet important sur la fin de la guerre ${ }^{8}$.

Ce même jour, le Régiment recevait la visite de deux correspondants de guerre, l'un français, l'autre américain. Ce dernier, s'entretenant avec un officier du Q.G. régimentaire, lui dit: "I hear you throw big stuff around this joint. Could I have a look at 'em guns ?" Il raconta ensuite qu'il avait déjà relaté les exploits du Régiment, autour de Vaucelles et de Falaise, surtout les événements dramatiques du 8 août, dans un journal américain ${ }^{9}$.

\section{MESSAGE DU GÉNÉRAL CRERAR}

La veille de l'offensive, le 7 février, le général Crerar adressait le message suivant à l'armée canadienne. Sur la copie conservée dans les archives du brig. Gagnon, on peut lire que ce message devait être communiqué à tous les membres du Régiment et détruit ensuite par le feu avant 11 h. 59 ce soir-là :

Il y a six mois, presque jour pour jour, les formations de cette armée ont écrit une page d'histoire, lors de leur poussée vers le Sud, qui a permis de rompre la "charnière de Caen" et de fermer la "poche de Falaise". Ces mêmes formations, ainsi que d'autres, enregistreront de nouveau d'autres grands succès,

7 Ibid., 490.

8 Journal de guerre du Régiment, XXXV: Ordres du jour, 3.

9 Archives de l'auteur [Nimègue], 6 février 1945. 
dans les jours qui vont suivre, en enfonçant le "pivot" du Reichswald et en tournant ce secteur-clé de la ligne Siegfried.

Les opérations que nous allons bientôt entreprendre sont de la plus haute importance. A vrai dire, leur résultat pourrait amener une victoire rapide et complète des Alliés. L'assaut sera lancé en grande force et soutenu par un feu des plus puissants. Quelles que soient les difficultés du terrain et du temps, la poussée à travers l'ennemi et ses défenses se poursuivra sans répit. Il faut qu'il n'ait ni le temps ni l'occasion de réfléchir ou de rassembler ses ressources. L'occasion s'ouvre devant nous. Voyons à la saisir fermement et à l'exploiter d'une façon décisive ${ }^{10}$.

\section{LE RÔLE CAPITAL DE L'ARTILLERIE}

Pour assurer la réussite de cette opération importante, le lieutenant-général Brian Horrocks, commandant le $30^{\mathrm{e}}$ corps britannique (ce dernier devant effectuer la trouée initiale) avait exigé que les troupes d'assaut reçoivent "l'appui d'une artillerie formidable". En fait, le plan de tir prévoyait un "bref mais formidable" bombardement destiné à annihiler toute résistance ennemie à l'assaut initial, à saturer complètement les défenses allemandes et à détruire ou réduire à l'impuissance les casemates bétonnées de l'ennemi ${ }^{11}$.

Du 25 janvier au $1^{\text {er }}$ février, 500,000 obus de 350 pièces différentes, pesant 11,000 tonnes, avaient été transportés aux emplacements des canons. Or, sept jours avant l'offensive, 1,034 canons de tous calibres, y compris 300 moyens, lourds et superlourds, n'étaient pas encore rendus à leurs places respectives, et cela pour ne pas alerter l'ennemi. Toute erreur de distribution des obus aurait pu éliminer des batteries complètes. Mais il n'y eut pas d'erreur ${ }^{12}$ ! L'artillerie avait une telle importance dans anglais).

10 Archives du brigadier J.-H.-R. Gagnon, OBE (traduit de l'original

11 Stacey, op. cit., 492.

12 Thompson, op. cit., 145. 
cette opération que toute erreur eût été impardonnable. Montgomery devait écrire plus tard:

The main features of the break-in operation were to be the development of a tremendous weight of artillery from well over a thousand guns... The effects of our bombardment were very considerable, and in particular the counter battery measures largely neutralized the ennemy artillery ${ }^{13}$.

Jusqu'à quel point une telle préparation d'artillerie étaitelle nécessaire ? Pour s'en rendre compte, il suffit de savoir que toute la région comprise entre Clèves et Goch, - ces deux localités constituant les charnières de deux mâchoires étroites mais puissantes, - devait être enfoncée, face aux meilleures troupes ennemies encore disponibles dans l'Ouest, à savoir la $1^{\text {re }}$ armée de parachutistes du groupe d'armées $H$. Ces troupes, - parachutistes de nom seulement, - n'en étaient pas moins très bien entraînées, jeunes et résolues à défendre chaque pouce du territoire allemand. La $84^{\mathrm{e}}$ division défendait la forêt du Reichswald et, avec les $180^{\mathrm{e}}$, $190^{\mathrm{e}}$ et $606^{\mathrm{e}}$ divisions en réserve immédiate, était soutenue par environ 114 canons ${ }^{14}$.

\section{DÉCISION IRRÉVOCABLE DU MARÉCHAL MONTGOMERY}

Le lieutenant-général Horrocks, de son côté, disposait de 10 divisions, de 5 groupes d'artillerie, de 1,000 bombardiers lourds et des appareils de la $2^{\text {e }}$ force aérienne tactique de la R.A.F., ainsi que des bombardiers lourds et moyens des $8^{\mathrm{e}}$ et $9^{\mathrm{e}}$ forces aériennes des Etats-Unis. Tout cela, affirme Thompson, dans son ouvrage sur la bataille de la Rhénanie:

... together with the opening barrage or more than 1,000 guns, to which would be added the fire pattern of three machine gun battalions and the $8^{\text {th }} \mathrm{Ar}$ moured Brigade's tanks, revealed Feld Marshall Montgomery's intention to shoot his way into the battlefield, and to force the jaws closing the way to the Rhine ${ }^{15}$.

${ }^{13}$ Feld maréchal Montgomery, Normandy to the Baltic, (Londres, Arrow Books, 1961), 188-189.

14 Thompson, op. cit., 151.

15 Ibid., 152. 


\section{DU CÔTÉ DE L'ENNEMI}

On a peine à croire que l'ennemi ignorât ce qui se préparait contre lui. Pourtant, à l'aube du 8 février, malgré un bombardement aérien sur Clèves, la veille, on rapporte que le général Schlemm fut "éveillé par le grondement d'une canonnade sans précédent sur le front de l'Ouest". "Je sens la grande offensive", déclara-t-il 16. Il "n'avait jamais entendu quelque chose de pareil sauf dans les très grandes batailles du front russe ${ }^{17 "}$.

En fait, il s'agissait du plus puissant barrage d'artillerie de toute la guerre, de la part des alliés occidentaux. Voici ce qu'en dit Thompson, qui en fut témoin:

The effect on those who lay or moved under the guns was awe-inspiring, constraining a new awareness, a tentative appraisement of what might lie ahead. Impossible to imagine the effect upon the enemy, as the air vibrated with incessant thunder to deaden the senses, and the earth shuddered ... All the wild pattern of the sky kindled the grey water to the semblance of a boiling cauldron of fire and flame. Soon it was possible to distinguish the instruments in the vast uproar, the heavies and mediums in layers above the threshing and burping of the smaller weapons. Underneath the great weight of the barrage the Bofors were firing flat while the tanks of the armoured brigades and the machine gunners of three battalions drenched the forward positions of the enemy ${ }^{18}$.

Toujours au sujet de la violence du tir d'artillerie du 8 février, le colonel Stacey déclare, pour sa part, que "la concentration du bombardement des positions de la $84^{\mathrm{e}}$ division allemande dépassa probablement en violence toute autre opération du genre sur un front aussi limité, au cours de toute la campagne

16 Georges Blond, L'Agonie de l'Allemagne (1944-1945), (Ottawa, Cercle du Livre de France, 1953), 198.

17 Major M. Shulman, La Défaite allemande à l'Ouest, traduit de l'anglais par le capitaine de corvette André Cogniet (Paris, Payot, 1948), 310.

18 Thompson, op. cit., 161. 
occidentale". Il ajoute qu'en moyenne plus de 9 tonnes d'obus tombèrent sur chacune des 268 cibles choisies ${ }^{19}$.

\section{LE RÔLE DU REGIMENT}

Dès 5 h. du matin, le 8 février, le Régiment ajoutait le grondement sinistre de ses seize pièces à ce tintamarre infernal. Il s'agissait pour lui de neutraliser l'artillerie ennemie. Vers 10 heures du matin, il participait à un barrage en soutien du $30^{\mathrm{e}}$ corps d'armée britannique, qui devait durer jusqu'à $1 \mathrm{~h}$. de l'après-midi.

A 5 h. de l'après-midi, il ouvrait le feu sur le front de la $3^{e}$ division canadienne, en soutien des $7^{\mathrm{e}}$ et $8^{\mathrm{e}}$ brigades canadiennes. De tous côtés, on rapportait que le tir du Régiment était très précis, et que le moral ennemi était fort ébranlé. Le major Tremblay et le capitaine Sévigny, de retour au Q.G. régimentaire à 8 heures du soir, rapportaient que le tir du Régiment avait été un "real good show ${ }^{20 " . ~ E n ~ f a i t, ~ c o m m e ~ l e ~ r a c o n t e ~ T h o m p s o n: ~}$

When the barrage lifted from its opening phases... and the forward troops advanced on its heels through the minefields, it seemed that no man could have lived in the enemy positions, that no tree could still be left standing in all the Reichswald forest ${ }^{21}$.

Et un officier du Régiment, faisant part de ses impressions du 8 février, écrivait à son frère, deux jours plus tard:

J'ai hâte de te raconter... les détails du plus formidable barrage d'artillerie auquel j'ai participé au début de cette offensive. Même El-Alamein est un feu d'artifice, comparé à ce que nous avons craché ici ${ }^{22}$.

\section{REACTION DE L'ENNEMI}

Pourtant, malgré l'extrême violence de ce tir préparatoire d'artillerie, il est étonnant de savoir que l'ennemi pût réagir si

19 Stacey, op. cit., $494-495$.

20 Journal de guerre du Régiment, XXXV : 4.

21 Thompson, op. cit., 162.

22 Archives de l'auteur [Nimègue], 10 février 1945. 
bien ${ }^{23}$. En effet, même si, le soir du 8, l'ennemi avait encaissé 500,000 obus ${ }^{24}$, il était clair, deux jours plus tard, que l'ennemi était résolu à combattre farouchement pour défendre chaque pouce du sol allemand, à l'ouest du Rhin. D'autant plus que le moral allemand restait élevé et qu'il n'y avait pas lieu de sous-estimer l'esprit combatif d'un ennemi décidé à tenir jusqu'au bout en deçà du Rhin ${ }^{25}$.

Malgré cela, dès le 10 février, la moitié de la forêt du Reichswald était libérée et Clèves tombait, bien que, sur le front de la $3^{\mathrm{e}}$ division canadienne, les troupes fussent dans trois pieds d'eau et dussent avancer au moyen de véhicules amphibies ${ }^{26}$. Même les artilleurs eurent du mal à avancer, sur les traces des fantassins, d'autant plus qu'il restait encore des poches de défenseurs allemands non éliminées par l'infanterie ${ }^{27}$.

A la fin de ce même 10 février, l'opération "Veritable" était devenue un enchevêtrement tactique impossible à décrire, et on ne saurait en retracer le déroulement qu'en recourant à une masse de documents. A vrai dire, même les archives des corps d'armée, voire des divisions, ne sauraient nous apprendre grand'chose; d'où l'utilité, espérons-nous, d'une histoire régimentaire, car, en réalité, c'est à ce niveau que se déroulent les batailles ${ }^{28}$.

Le 11 février, le Régiment tirait sur quelques cibles, toutes au nord du Rhin, alors que le maréchal Montgomery, en visite sur le front anglo-canadien, paraissait satisfait de la situation ${ }^{29}$.

\section{L'ARTILLERIE BRISE LES CONTRE-ATTAQUES}

C'est à ce moment-là, soit vers le 11 ou le 12 février, que le tir précis de l'artillerie se révéla essentiel pour briser les furieuses contre-attaques de l'ennemi ${ }^{30}$. On apprenait, en effet, que

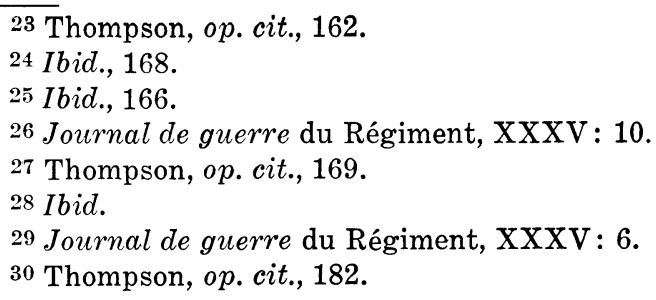


les troupes anglo-canadiennes progressaient de façon satisfaisante malgré des contre-attaques au sud-est de la forêt du Reichswald et au sud de Clèves. Même si le Régiment, pour sa part, ne pouvait avancer encore, à cause de l'encombrement des routes, on apprenait officiellement que la première ville capturée en Allemagne par la $1^{\text {re }}$ armée canadienne était Wyler. Et c'est au Régiment de Maisonneuve, de Montréal, qu'échouait cet honneur ${ }^{31}$.

\section{LA MASCOTTE DU RÉGIMENT}

Le 13 février, la première phase de l'opération "Veritable" était terminée. Ce même jour, se produisait au Régiment un événement de la plus haute importance. En effet, "Brigitte", la chienne du "padre", disparue avant Noël, était retrouvée près de Wyler ${ }^{32}$. Voici comment un officier décrivait cet événement quelques jours plus tard:

Le plus grand événement du Régiment, ces jours-ci, c'est le retour d'un de nos enfants prodigues: "Brigitte", la chienne du "padre" ! Cette chienne est devenue, comme mon "batman" Jos. Ouellet, une sorte d'institution légendaire. En effet, le "padre" traîne cette "Brigitte" depuis deux ans, après l'avoir adoptée quelque part en Angleterre. Elle a participé à toutes les manœuvres en Angleterre et elle a fait toute la campagne avec nous, suivant le "padre" partout et toujours. Il y a deux mois, elle disparut soudainement, et le "padre" en fut très affligé. Une annonce avec récompense fut mise dans le journal local (en anglais et en hollandais) afin de retrouver la brebis égarée. Or, voici qu'il y a deux jours, le "padre" la retrouvait dans un autre régiment. Ce fut une fête au mess ce soir-là... Malgré ses campagnes exténuantes, elle a réussi à accoucher de sa sixième portée, étant mère, à date, de 24 petits chiens! Quand nous étions à Boulogne, le "padre" traînait encore avec lui une boîte avec ses quatre derniers !... De retour au bercail, nous avons tué le veau gras (lequel, en l'occurrence, est du "bully beef"') et tout le monde est heureux ${ }^{33}$.

31 Journal de guerre du Régiment, XXXV: 6.

32 Ibid., 7.

33 Archives de l'auteur [Nimègue], 13 février 1945. 
Le "padre", rassuré par le retour de sa "Brigitte", continua de s'adonner de plus belle à sa nouvelle passion: le bridge. On lit, en effet, dans cette même lettre:

A part cet événement capital [le retour de "Brigitte"], rien de spécial ne vient déranger notre partie de bridge quotidienne. Le "padre", ayant assimilé toute une bibliothèque "culbertsonienne", entasse les francs, les guilders et les florins. Son grand argument pour excuser son gain est celui-ci: "Ça vous en fera moins pour le whiskey ${ }^{34}$ '”

\section{LES CONTRE-ATTAQUES ENNEMIES SONT REPOUSSÉES}

Le 14 février, on enregistrait encore de nombreuses contreattaques ennemies sur tout le front. Elles furent toutes repoussées mais, d'autre part, il n'y eut aucune avance du côté des Alliés ${ }^{35}$. En fait, outre des conditions atmosphériques défavorables, deux autres facteurs empêchaient la rapide exploitation de l'offensive: le fanatisme et l'obstination des parachutistes allemands, et l'habileté du général Schlemm. A ce propos, le colonel Stacey écrit:

La résistance opposée par les divisions parachutistes allemandes fut plus farouche et plus inflexible que tout ce que l'on avait vu jusque-là dans l'Ouest ${ }^{36}$.

C'est ce que confirme le général Eisenhower en écrivant que: "L'opération du nord s'est heurtée à la résistance la plus acharnée que l'ennemi nous ait opposée n'importe où le long du fleuve ${ }^{3 \tau}$." Et le maréchal Montgomery devait, pour sa part, écrire: "... the German parachute troops fought with fanatical obstinancy and ferocity and, however untenable the situation, hung on to the last man ${ }^{38}$ ".

Le 15 février, on avait réussi à identifier neuf divisions ennemies sur le front du $21^{\mathrm{e}}$ groupe d'armées: 1 Panzer, 1 Pan-

34 Ibid.

35 Journal de guerre du Régiment, XXXV: 7.

36 Stacey, op. cit., 312-314.

37 Ibid., 566 (cité).

38 Montgomery, op. cit., 192. 
zer de grenadiers, 3 d'infanterie et 4 de parachutistes. Ces neuf divisions étaient soutenues par une artillerie redoutable, des canons autopropulsés et des mortiers. Même si l'artillerie ennemie ne comptait qu'un dixième environ de la puissance de feu de l'artillerie anglo-canadienne, elle n'en réussit pas moins à opposer un feu des plus nourris aux assaillants ${ }^{39}$.

\section{PROGRESSION DES CANADIENS}

Le 16 février, comme le temps s'était mis au beau, les chasseurs et bombardiers alliés se mirent à pilonner les communications, les points fortifiés et les ponts ennemis. Ce qui permit sans doute à la 3e division canadienne de progresser vers Calcar, bien que l'ensemble du front ouest fût relativement calme ce jourlà. Par ailleurs, on rapportait que les Russes approchaient de Dresde, à 90 milles au sud de Berlin. Ce même jour, deux correspondants de guerre, l'un canadien, l'autre français, interviewaient des officiers et des canonniers de la $58^{\mathrm{e}}$ batterie ${ }^{40}$.

\section{LE RÉGIMENT ENTRE EN ALLEMAGNE}

Le lendemain, 17 février, le Régiment recevait enfin l'ordre d'avancer. A $4 \mathrm{~h}$. de l'après-midi, il entrait en Allemagne, au nord de Gennep, pour soutenir la $2^{\mathrm{e}}$ division canadienne qui devait se lancer à l'attaque le lendemain, entre la $15^{\mathrm{e}}$ division britannique et la $3^{\mathrm{e}}$ division canadienne. L'objectif de cette attaque était le terrain élevé à l'est d'Udem. Le Q.G. régimentaire et les P.C. des deux batteries s'installaient dans Clèves, alors que le capitaine Yvon Thériault se rendait au Q.G. de la $2^{\mathrm{e}}$ division, comme représentant du commandant ${ }^{41}$.

Goch était en ce moment l'enjeu de la bataille, qui fut extrêmement sanglante. Voici comment Thompson décrit la scène qui se présentait le 18 février:

The trail of war was increasingly marked by the roasted bodies of men in armour, men in aircraft,

39 Thompson, op. cit., 187.

40 Journal de guerre du Régiment, XXXV: 8.

41 Ibid. 
men in pill boxes, men in cellars, their charred torsos swollen, rigid and terrible to see. And it seemed that the stench of burning flesh was as clinging in the nostrils as that of burning rubber ${ }^{42}$.

Mais ce n'était pas encore la fin, loin de là. Ce même 18 février, le Régiment ouvrait le feu pour la première fois en Allemagne, à 11 h. 59 du soir ${ }^{43}$. Cette même date, un officier du Régiment écrivait ce qui suit:

Enfin, nous sommes entrés dans le cœur de la forteresse hitlérienne... La fameuse "festung Europa" est définitivement enfoncée et vouée à la destruction la plus inouie. Jusqu'ici, je n'ai vu que des ruines indescriptibles ... Hier, j'ai vu quelques civils allemands pour la première fois. Leur regard était morne, presque sinistre; la plupart détournaient les yeux pour ne pas me voir. C'est un mélange de haine et de lassitude ${ }^{44}$.

Et voici, dans une autre lettre datée du même jour, la description de Clèves après le bombardement aérien préalable à l'offensive du 8 février:

... il est impossible de te donner une idée de ce qu'est une ville bombardée par 1,000 bombardiers lourds en une seule nuit! C'est un amas de décombres, de fer tordu, de pianos éventrés, de vêtements éparpillés jusque dans la rue; enfin, c'est une scène lamentable à voir. Et, à travers ce tableau, tu peux voir des civils, l'air hagard, allant je ne sais où ... Tu n'as pas à t'inquiéter, les Allemands sont bel et bien "kaput", c'est-à-dire f . . ., écrasés, battus comme des chiens. Par un entêtement imbécile, Hitler semble vouloir que tout périsse avec lui : voilà pourquoi la guerre dure toujours... Je suis entré en Allemagne, non avec un sentiment de haine, mais avec la détermination d'en finir au plus tôt ${ }^{45}$.

Oui, la guerre durait toujours et semblait même devenir de plus en plus féroce. On peut lire, en effet, dans le Journal de

42 Thompson, op. cit., 194.

43 Journal de guerre du Régiment, XXXV : 8.

44 Archives de l'auteur [Clèves], 18 février 1945.

45 Ibid., autre lettre, même date. 
guerre du Régiment, en date du 19 février: "Quelle journée ! Attaques, contre-attaques, duels d'artillerie, et encore d'autres contre-attaques ${ }^{46} . "$ Le Régiment devait, en effet, ce jour-là, exécuter un plan de tir en soutien de la $7^{\mathrm{e}}$ brigade canadienne, dans le cadre de l'attaque de la $2^{\text {e }}$ division, prévue à midi après un barrage préliminaire. Entre-temps un canon de $88 \mathrm{~mm}$ ouvrait le feu sur le Régiment, pour y incendier plusieurs véhicules et mettre le feu aux munitions. Ce fut un véritable feu d'artifice qui, heureusement, ne causa aucune perte humaine. Les canons du Régiment tirèrent quand même sans arrêt, et "nos troupes avancées, soutenues par un tir précis et rapide pour enrayer les contre-attaques ennemies, les repoussent toutes ${ }^{47}$ ". C'est ce qui faisait sans doute écrire à un officier du Régiment, ce jour-là: “.... je t'assure que les Boches attrapent en ce moment ce qu'on appelle en bon "canayen" une "gratte 48 ".

\section{HÉROÏSME D'UN OFFICIER DU RÉGIMENT}

Le 20 février allait se révéler une journée extrêmement mouvementée. D'abord, tôt le matin, la $4^{\mathrm{e}}$ brigade canadienne comptait deux de ses bataillons complètement encerclés par l'ennemi: le Royal Hamilton Light Infantry, auquel était attaché le capitaine Poulin, et l'Essex Scottish, auquel était attaché le capitaine Thériault. A midi, l'Essex Scottish était considéré comme perdu. Au Régiment, on ne recevait aucune nouvelle du capitaine Thériault depuis quinze heures. A son retour, il raconta qu'obligé de se réfugier dans une cave, il avait fait détruire deux chars allemands par les canons du Régiment, alors que ces chars se trouvaient exactement au-dessus de lui. Il ajouta qu'il avait été encerclé pendant des heures par des chars Tigre et des fantassins allemands. L'atmosphère était devenue si tendue dans cette cave, où se trouvaient avec lui d'autres officiers canadiens, qu'à un moment donné, un officier canadien-anglais, pris de panique, voulut donner le signal de la reddition. Le capitaine Thériault, toujours flegmatique, eut la présence d'esprit de

46 Journal de guerre du Régiment, XXXV : 9-10.

47 Ibid.

48 Archives de l'auteur [Clèves], 19 février 1945. 
réduire au silence ce malheureux officier, épargnant ainsi la capture de tous ceux qui étaient cachés avec lui dans cette cave. On comprendra d'autant mieux le cran extraordinaire du capitaine Thériault en disant que, peu de temps avant, il avait abattu à bout portant, au revolver, un Allemand qui avait eu la malencontreuse idée de se montrer à un soupirail de cette cave ${ }^{49}$. Pendant que se déroulaient ces scènes dramatiques à l'avant, le Régiment ne cessait de tirer: 1,600 obus dans la journée, dont 40 p. 100 entre 5 et 7 heures du soir, alors que les Allemands contre-attaquaient sans cesse avec des chars et des fantassins. Le lieut. Racine, de retour du Q.G. de la $2^{\mathrm{e}}$ division canadienne, où il était officier de liaison ce jour-là, raconta comment chacun là-bas était heureux de l'appui du Régiment. En effet, le Régiment fut constamment le premier à tirer parmi quelques douzaines d'autres, même des régiments de campagne, dont les pièces étaient plus faciles à manœuvrer. Tous les appels de soutien furent répondus en moins de deux minutes, toute la journée ${ }^{50}$.

\section{LE SECTEUR NORD DE LA LIGNE SIEGFRIED S'EFFONDRE}

Avec la chute de Goch, le 21 février, le dernier grand bastion des principales défenses de la ligne Siegfried dans le Nord s'était écroulé. Malgré cela, on s'étonne que l'ennemi pût encore soutenir le combat contre les bombardements aériens incessants, les fusées des Typhoon et le tir des mitrailleuses, sans compter le feu constant de l'artillerie ${ }^{51}$. Pour sa part, le Régiment avait tellement mis ses canons à contribution que, le 21 février, dans une note adressée aux commandants de batterie, le lieut.-colonel Gagnon affirmait que 8 canons sur 16 avaient besoin d'être vérifiés, quant à la précision de leur tir, à cause de leur usure ${ }^{52}$.

Vers la fin de l'après-midi de ce même jour, les troupes canadiennes étaient rendues aux abords de Moyland, emplacement fortifié sur la route Clèves-Colcar. Les capitaines Giroux et Chabot remplaçaient, ce jour-là, le major Lahaie et le capitaine

49 Journal de guerre du Régiment, XXXV : 10.

50 Ibid.

51 Thompson, op. cit., 196.

52 Archives du brigadier J.-H.-D. Gagnon, 21 fóvrier 1945. 
Poulin à l'avant. Ce dernier eut beaucoup à raconter à propos de son encerclement avec le Royal Hamilton Light Infantry. Il connut en effet des aventures analogues à celles du capitaine Thériault, mais malheureusement aucun document n'en fait état ${ }^{53}$.

\section{DES CANADIENS FRANÇAIS CHEZ FRÉDÉRIC LE GRAND}

Le 22 février, la chute du château de Moyland, aux mains des troupes canadiennes-françaises, marquait la fin de la deuxième phase de la bataille de la Rhénanie. Voici ce qu'écrit Thompson à ce propos:

... it seemed to mark also the end of an era, an anachronism in its setting... In the great principal chamber [du château], the curving walls embellished with paintings of flamboyant nudes, the cooking stoves of a French Canadian company roared under pans of frying fat. Here Frederick the Great, at ease in the canopied bed on the raised dais, had once hold the morning levee ${ }^{54}$.

Le 22 février, alors que la R.A.F. pilonnait les positions ennemies, le Régiment se préparait à occuper une nouvelle position, en vue d'une autre grande opération ${ }^{55}$. Dans la matinée et dans l'après-midi du 23, le Régiment exécutait un plan de tir à l'appui de la $15^{\mathrm{e}}$ division britannique (Scottish) qui attaquait au sud-est de Goch, en direction d'Udem. Et, peu après souper, le lieutenant Malouin allait chercher au Q.G. du $2^{\mathrm{e}}$ groupe un autre plan de tir, destiné cette fois à appuyer la $52^{\mathrm{e}}$ division britannique ${ }^{56}$.

\section{L'ENFER DE LOUISENDORFF}

Le lendemain, 24 février, allait être une autre journée mémorable. Le Régiment, parti dans la matinée pour occuper sa nouvelle position, était en batterie à $11 \mathrm{~h}$. Soudain, toute la position du Régiment fut systématiquement arrosée d'obus de canons et de mortiers ennemis. Cet enfer dura de 3 à 4 heures, au cours

\footnotetext{
53 Journal de guerre du Régiment, XXXV : 11.

54 Thompson, op. cit., 197.

55 Journal de guerre du Régiment, XXXV : 11.

56 Ibid., 12.
} 
desquelles le Régiment enregistra dix pertes: le capitaine Sévigny, grièvement blessé, dut se faire amputer la jambe gauche. Neuf canonniers, dont 3 morts (Lamirande, Gosselin et Ruest) allaient grossir le chiffre des pertes. Sans le savoir jusque-là, le Régiment était en batterie à environ 1,500 verges des lignes avancées ennemies. Il fallut l'intervention des Typhoon de la R.A.F. pour mettre fin à ce carnage. Malgré cela, les capitaines Dupuis et Thériault se rendaient, le jour même, au Q.G. de la $4^{\mathrm{e}}$ division blindée canadienne, en qualité d'officiers observateurs en vue de l'opération "Bluckbuster", prévue pour le surlendemain ${ }^{57}$.

\section{LE JOURNAL DE L'ARMÉE CANADIENNE PARLE DU REGIMENT}

A cette même date paraissait, dans le Maple Leaf (journal de l'Armée canadienne), dans la section réservée au Canada français, les lignes suivantes, signées du correspondant de guerre Jean-Charles Daoust:

Souvent nous avons parlé des exploits d'un régiment canadien-français d'artillerie, sans jamais toutefois le désigner. Le censeur nous permet enfin, pour la première fois depuis son débarquement en Normandie, de nommer le $4^{\text {e }}$ Régiment d'artillerie moyenne.

Ce régiment, composé presque entièrement de Canadiens français, s'était fait remarquer en Angleterre par la précision de son tir puisqu'il remporta les honneurs dans plusieurs concours.

Le $4^{\mathrm{e}}$ Régiment, m'écrit le lieutenant Phil Lauzon [autre correspondant de guerre], possède de nombreux amis chez les commandos français et britanniques qui débarquèrent à Flessingue (Walcheren). Récemment, le chef des commandos français m'exprima toute son admiration à l'égard de ce régiment d'artillerie canadien-français et particulièrement pour le capitaine Moss, de Montréal, qui était, au moment du débarquement à Flessingue, officier observateur d'artillerie avec la première vague d'assaut des commandos.

57 Ibid. 
Ma visite à ce régiment [poursuivait le lieutenant Lauzon], qui s'est signalé à Caen et à Falaise, s'est terminée d'agréable façon. Le major Maurice Archer, de Québec, m'invite à prendre le thé dans son abri souterrain. J'y rencontre le capitaine Luc Chabot, de Kingston, et le capitaine Jean-Pierre Giroux, de Québec. Pendant que nous bavardons et que nous essayons d'équilibrer, avec deux mains seulement, une tasse, une soucoupe, un gâteau, une cigarette, un crayon et un calepin, nous entendons la merveilleuse symphonie de tous les canons du Régiment, crachant leur acier sur l'Allemagne. C'est une musique bien douce à entendre, surtout que maintenant ces obus ne tombent plus en France, en Belgique, ni en Hollande, mais en Allemagne ${ }^{58}$.

\section{AUTRE OPERATION DE GRAND STYLE}

Cette "symphonie" n'en était pas encore à son dernier "mouvement", loin de là. En effet, dès le lendemain, 25 février, tous les P.C. du Régiment étaient occupés à préparer les plans de tir de l'opération "Blockbuster", prévue pour 4 h. 30 le lendemain matin. Cette nouvelle opération, la plus considérable jamais montée par un corps d'armée canadien, commandé par le lieutenant-général Simonds, allait faire appel à 40 régiments d'artillerie, aux $2^{\mathrm{e}}, 3^{\mathrm{e}}$ et $4^{\mathrm{e}}$ divisions canadiennes, à la $11^{\mathrm{e}}$ division blindée des Gardes (britannique), et à la $43^{\mathrm{e}}$ division britannique en réserve. Pour cette opération, le capitaine Giroux était attaché à la $6^{\mathrm{e}}$ brigade canadienne, le capitaine Moss à la $2^{\mathrm{e}}$ division canadienne, le capitaine Dupuis à la $10^{\mathrm{e}}$ brigade canadienne, et le capitaine Thériault à la $4^{\mathrm{e}}$ brigade blindée canadienne ${ }^{59}$.

\section{RESISTANCE OPINIÂTRE DE L'ENNEMI}

Dès l'aube du 26, le Régiment ouvrait le feu, quarante-cinq minutes avant l'heure $\mathrm{H}$. La $2^{\mathrm{e}}$ division canadienne progressa de façon satisfaisante sur le terrain protégé par le barrage. A la fin de la journée, Udem était atteint, ainsi que le chemin de fer au sud de la forêt du Hochwald ${ }^{60}$. Mais ce ne fut pas sans

58 Archives de Jean-Charles Daoust, 24 février 1945.

59 Journal de guerre du Régiment, XXXV : 13.

60 Ibid. 
une résistance opiniâtre de l'ennemi. En effet, voici ce qu'on peut lire dans l'ouvrage de Thompson, sur la situation le 27 février:

The enemy did not give the impression of a brittle crust which might crack wide open. It was hard right through ... ${ }^{61}$.

For days and nights the enemy had been battered mercilessly by artillery and aircraft, denied all hope of relief, of rest, of victory, or even of escape. It was astonishing, not that some were 'punch druk' or exhausted, but that so many continued to fight with a desperate and fanatical violence in the last flare-up of their nervous energies ${ }^{62}$.

\section{VISITE AU RÉGIMENT D'OFIICIERS RUSSES}

Malgré cette résistance farouche, tant sur le front ouest que sur le front est, on sentait, vers la fin de février, que l'Allemagne nazie vivait ses derniers jours, voire ses dernières heures. On en avait un indice assez concret, le 27 février, lorsque deux officiers de l'Armée rouge rendirent visite au Régiment, ce qui fit dire au "batman" Jos Ouellet: "J'savais pas que les Russes étaient si proches ${ }^{63}$ !" $\mathrm{Au}$ fond, dans toute sa naïveté, cette réflexion n'était pas tellement éloignée de la vérité. Dans un secteur plus immédiat, on s'attendait même à la jonction prochaine de la $9^{\mathrm{e}}$ armée américaine avec la $1^{\text {re }}$ armée canadienne ${ }^{64}$.

\section{PILLAGE ET FIN IMMINENTE DE LA GUERRE}

Grâce à l'écroulement de l'Allemagne, les gars du Régiment ne se faisaient guère plus de scrupule pour améliorer leurs rations alimentaires plutôt monotones par le moyen classique du pillage pur et simple, comme en fait foi cet extrait de lettre d'un officier du Régiment, en date du 28 février:

A la lueur d'une chandelle, du fond de mon trou, je t'envoie ces quelques mots. Nous mangeons très bien

61 Thompson, op. cit., 209.

62 Ibid., 212.

63 Journal de guerre du Régiment, XXXV : 13-14.

64 Ibid. 
depuis que nous sommes en Allemagne, car nous ne nous faisons aucun scrupule de faire boucherie avec les bœufs et les cochons qui nous tombent sous la main ... je devrais dire sous le pistolet ${ }^{65}$.

On commençait aussi à parier sérieusement sur la fin imminente de la guerre. On peut lire, en effet, dans cette même lettre:

Depuis quelque temps, il se fait de gros paris au Régiment au sujet de la fin de la guerre. Les optimistes placent la fin au mois de mars, et les plus sages au mois de juin ${ }^{66}$.

\section{LE COMBAT CONTINUE DE FAIRE RAGE}

$\mathrm{Au}$ cébut de mars 1945, alors que les premiers éléments du $1^{\text {er }}$ corps d'armée canadien arrivaient d'Italie dans le nord-ouest de l'Europe pour grossir les rangs de la $1^{\text {re }}$ armée canadienne, le combat continuait encore de faire rage. En effet, le $1^{\text {er }}$ mars, les capitaines Thériault et Dupuis, revenus exténués de leur mission à l'avant, rapportaient en avoir vu de toutes les couleurs. Le capitaine Thériault raconta notamment que le char dans lequel il voyageait fut frappé deux fois par des obus ennemis et qu'à une occasion il vit une forte contre-attaque se diriger vers lui. Or, le tir du Régiment attrapa les Allemands juste au moment où ils s'apprêtaient à avancer.

Il raconta en outre qu'un soir une patrouille ennemie entoura son char et chercha à le détruire à la grenade. Encore une fois, le tir du Régiment sauva le capitaine Thériault de cette situation périlleuse ${ }^{6 \tau}$.

De nouveau, le $1^{\text {er }}$ mars, l'aviation alliée pilonnait, par erreur, les lignes anglo-canadiennes, à 2,000 verges du Régiment, déclenchant ainsi des incendies qui durèrent pendant des heures ${ }^{68}$.

65 Archives de l'auteur [Louisendorff], 28 février 1945.

66 Ibid.

67 Journal de guerre du Régiment, XXXVI: 1.

68 Ibid. 


\section{IMMINENCE D'UNE JONCTION CANADO-AMÉRICAINE}

Le grand événement du 2 mars fut la nouvelle, reçue vers 7 heures du soir, selon laquelle les Américains avaient fixé une ligne d'arrêt du tir, afin d'éviter tout incident mortel entre les troupes canadiennes et les troupes américaines, alors sur le point d'établir leur jonction sur le Rhin. En effet, après avoir capturé Venlo, les blindés américains, progressant rapidement, ne se trouvaient plus qu'à moins de 10 milles des éléments canadiens. Sur le front $d u 30^{\mathrm{e}}$ corps britannique, l'ennemi était également en retraite, et Weeze tombait aux mains des alliés. Poussant plus loin vers le Sud, ces derniers entraient ensuite dans Kevelaer. D'autre part, l'ennemi résistait encore obstinément sur le front du $2^{\mathrm{e}}$ corps canadien, et d'âpres combats se déroulèrent encore. $\mathrm{La} 3^{\mathrm{e}}$ division canadienne, pour sa part, faisait parvenir au Régiment un plan de tir pour le lendemain, mais déjà les canons étaient hors de portée de l'ennemi ${ }^{69}$. Aussi, le major Archer dirigeait-il, dès le 3 mars, une nouvelle reconnaissance dans la région du sud-ouest de Calcar, non loin de l'extrémité occidentale de la forêt du Hochwald. Toute la journée, le Régiment attendit l'ordre d'avancer vers cette nouvelle position mais, à la fin de l'après-midi, on apprenait que cette avance était remise au lendemain matin à l'aube. Soudain, à 9 h. 30 du soir, l'ordre arrivait d'avancer. A 1 heure du matin, le Régiment était en batterie dans sa nouvelle position, à Todtenhuge. On apprenait alors que les Américains avaient fait la jonction avec les troupes canadiennes au nord de Geldern ${ }^{70}$. Les Allemands encore à l'ouest de cette nouvelle ligne établie par la jonction canado-américaine se trouvaient alors complètement encerclés. Mais ceux qui défendaient encore les approches de Xanten, sur le Rhin, se battaient encore désespérément ${ }^{71}$.

\section{LES ALLEMANDS TRAVERSENT LE RHIN}

Le 4 mars, à la faveur d'une pluie peu propice à l'activité aérienne, l'ennemi en profitait pour se retirer à l'est du Rhin en

${ }^{69} \mathrm{I} b i d$.

70 Ibid., 2, et Eisenhower, op. cit., 214.

71 Journal de guerre du Régiment, XXXVI : 2. 
toute vitesse, utilisant les quelques ponts restés encore intacts. Le Q.G. du Régiment se trouvait alors établi dans le sous-sol d'une vaste maison partiellement incendiée, à une croisée de chemins près de Todtenhuge, au sud-ouest de Calcar. En général, les opérations de nettoyage à l'ouest du Rhin se poursuivaient alors à un rythme satisfaisant, sauf autour de Xanten, où le gros des forces allemandes faisait face aux Canadiens. Plus au sud, la $1^{\text {re }}$ armée américaine du général Hodges s'approchait rapidement de Cologne, et la $3^{\text {e }}$ armée américaine du général Patton était sur le point de submerger les dernières défenses de la ligne Siegfried ${ }^{72}$.

Toutes ces bonnes nouvelles faisaient écrire ce qui suit à un officier du Régiment, ce même jour :

... Nous nous attendons à une autre poursuite sans arrêt, comme celle qui nous a mis sur les talons des Boches, à travers la France et la Belgique, après la bataille de Falaise. L'ennemi est épuisé, et nous tuons malheureusement des enfants en uniforme! Encore cet après-midi, j'ai contemplé avec tristesse deux adolescents, les yeux fixés vers le ciel, qui tombèrent, il ya quelques jours, sous le feu terrible de notre barrage ${ }^{\mathbf{7 3}}$.

Et, dans une lettre du 5 mars, de ce même officier, on peut lire:

Il n'y a pas de mots pour décrire la destruction qui règne ici. En ce moment, je suis dans une cave, car seules les caves sont habitables... Les champs autour de nous sont jonchés de vaches mortes et de débris de la déroute allemande. Le "padre" a enterré plusieurs cadavres allemands cet après-midi ${ }^{74}$.

\section{LES CHEVAUX REMPLACENT LES VEHICULES}

Pour ajouter à cette scène de désolation, un temps pluvieux rendait le terrain occupé par le Régiment absolument impraticable pour les véhicules, de sorte qu'il fallut recourir à des chevaux

72 Ibid.

73 Archives de l'auteur [Todtenhuge], 4 mars 1945.

74 Ibid., 5 mars 1945. 
pour vaquer aux échanges courants de communications entre les postes de commandement des batteries et le Q.G. régimentaire. Ainsi, le major Lahaie s'était-il muni d'un boghei, alors que ses estafettes transportaient ses messages à cheval. Le tir des canons n'en souffrait pas toutefois, puisque, le 5 mars, on rapporte que le lieutenant Lewis Martin, pour la première fois officier observateur à l'avant, ordonnait au Régiment de tirer quatre cibles "M" dans la même journée, c'est-à-dire des cibles nécessitant le tir de tous les canons à la fois. On peut lire dans le Journal de guerre du Régiment de ce jour-là: "De la façon dont vont les choses, il n'y aura plus un seul Allemand à l'Ouest du Rhin, sur notre secteur canado-américain, d'ici 48 heures ${ }^{75}$." De fait, le major de brigade du $2^{\text {e }}$ groupe informait le Régiment, le 6 mars, qu'il allait de nouveau avancer le lendemain, cette fois vers une position située à 1,500 verges seulement de l'ennemi ${ }^{76}$. La reconnaissance de cette nouvelle position s'effectua dans les lignes avancées, le Régiment ne devant s'y aventurer que sur un signal du major Archer. A 11 heures du matin, le Régiment s'ébranlait. Aussitôt, des obus ennemis se mirent à pleuvoir sur le convoi en marche. Le Régiment n'en parvint pas moins à s'établir aux environs d'un petit village, du nom de Labbeck.

Dans les champs environnants, on pouvait alors voir en position des mitrailleuses, des canons autopropulsés et des canons de DCA, le tout constamment en train de harceler les troupes ennemies ${ }^{77}$. On comprend que Schulman ait pu écrire, à propos du 8 mars 1945 :

...ces troupes, qui avaient lutté dans une bataille épuisante, sans le moindre répit pendant tout un long mois, étaient sans arrêt bombardées par des avions et par les masses de canons de l'inlassable artillerie alliée ${ }^{78}$.

On ne dira jamais assez, en effet, à quel point l'artillerie alliée a contribué à la défaite de l'Allemagne dans cette guerre.

75 Journal de guerre du Régiment, XXXVI: 3.

76 Ibid.

77 Ibid., 3-4.

78 Schulman, op. cit., 319. 


\section{LE RÉGIMENT FAIT OBSTACLE À LA RETRAITE DE L'ENNEMI VERS L'EST}

Le 8 mars encore, le Régiment s'éveillait le matin au son d'un violent tir de ses canons, qui ne dérougirent pas de la journée. A 9 heures du soir, un canonnier de la $58^{\mathrm{e}}$ batterie amenait au Q.G. régimentaire deux prisonniers déserteurs. Sur 22 membres de leur compagnie, racontèrent-ils, il ne restait plus que 4 survivants ${ }^{79}$.

Le 9 mars, dès 7 h. 15 du matin, le Régiment exécutait un nouveau plan de tir, cette fois à l'appui de la $43^{\mathrm{e}}$ division britannique (Welsh). Dans l'après-midi, le tir des canons était dirigé par un observateur aérien. Ce dernier rapporta par la suite que le tir avait été "marvellously effective, many fires observed and it is believed that many guns and vehicules were destroyed ${ }^{80}$ ".

Le lendemain, le Régiment engageait six batteries ennemies et, dans la matinée, deux gros navires allemands traversant le Rhin. Le tir sur ces bateaux était dirigé par un observateur aérien mais, malheureusement, bien que les obus du Régiment eussent failli les frapper, aucun ne réussit à les atteindre de plein fouet. Pourtant, dans l'après-midi, le tir reprit et, cette fois, l'observateur-pilote rapporta que le tir avait été "marvellous ${ }^{81}$ ".

L'ennemi, harcelé de tous côtés, en était enfin venu à la décision inévitable de se replier à l'est du Rhin. Pourtant, répondant à Rundstedt et à d'autres de ses généraux qui lui avaient demandé, peu de temps auparavant, de se replier à l'est du Rhin, Hitler avait répondu: "A quoi servirait de transférer une catastrophe d'un lieu à un autre ${ }^{82}$ ?" Cette fois, c'était bien la catastrophe finale. Et il n'est pas exagéré d'affirmer que l'artillerie alliée joua dans ce dénouement final un rôle de premier plan. Voici en effet ce qu'écrit Thompson, à propos de la période écoulée entre lo 6 et 10 mars 1945 :

\footnotetext{
79 Journal de guerre du Régiment, XXXVI: 4.

$80 \mathrm{Ibid}$.

81 Ibid., 5.

82 Thompson, op. cit., 5.
} 
...the pounding by artillery and bombing within the confines of that narrow triangle was aweinspiring ... Left, right and centre, the $43^{\text {rd }}$, the $52^{\text {nd }}$ and the $2^{\text {nd }}, 3^{\text {rd }}$ and $4^{\text {th }}$ Canadian Divisions, had gathered themselves for major attacks concentrated with all their strength, and supported by an immense weight of artillery ${ }^{83}$.

Du 8 février au 10 mars 1945, l'offensive anglo-canadienne avait infligé 100,000 pertes à l'ennemi, dont 50,000 prisonniers. Le 11 mars, les Américains, pour leur part, capturaient à eux seuls 50,000 autres prisonniers ${ }^{84}$.

\section{FIN DE LA BATAILLE DE LA RHÉNANIE}

C'est ce jour-là que la bataille de la Rhénanie prenait fin. Le $21^{\text {e }}$ groupe d'armées du maréchal Montgomery occupait désormais, pour sa part, toute la rive gauche du Rhin, de Nimègue à Düsseldorf ${ }^{85}$. A cette même date, le feld-maréchal Rundstedt était de nouveau limogé par Hitler, et remplacé par le feld-maréchal Kesselring, rappelé d'Italie à cette fin. "La dernière des véritables grandes batailles de la campagne... la fournaise de la Rhénanie ${ }^{86 "}$ était maintenant chose du passé.

\section{TÉMOIGNAGE DES GÉNÊRAUX}

Il ne restait plus désormais qu'à franchir le Rhin et opérer la jonction avec les Russes. Voici comment le général Eisenhower a décrit la bataille de la Rhénanie:

... les conditions atmosphériques auraient difficilement pu être plus défavorables ... Dans les premiers jours de février, le terrain se transforma en un véritable bourbier tandis qu'une grande partie de la zone où devait s'efiectuer notre avance était inondée. Les difficultés que nous eûmes à surmonter étaient immenses, et nos troupes durent quelquefois combattre avec de l'eau jusqu'à la ceinture. Elles endurèrent d'énormes privations, mais leur courage était

83 Ibid., 226-227.

84 Schulman, op. cit., 230.

85 Stacey, op. cit., 554.

sô Ibid., 557. 
indomptable et elles supportèrent leurs peines avec une grande bravoure en infligeant à l'ennemi une défaite très importante au cours de combats qui figurent parmi les plus violents de toute la guerre ${ }^{87}$.

Les troupes anglo-canadiennes, de leur côté, eurent à soutenir le fardeau le plus lourd de la résistance ennemie. En effet:

In the last stages of the battle, the enemy had mustered more than one thousand guns, and seven hundred and seventeen mortars, to subject the Canadians and British to the heaviest volume of fire experienced in the campaign ${ }^{88}$.

Ce fardeau plus lourd imposé aux troupes du maréchal Montgomery s'explique par le fait que:

... dans une situation remarquablement analogue à la bataille de Caen en Normandie, la crème des forces de von Rundstedt avait été attirée vers le Nord, dans le Reichswald où, pour continuer la métaphore, elle avait été sérieusement fouettée ${ }^{89}$.

C'est ce que confirme Thompson, dans son ouvrage sur la bataille de la Rhénanie, en écrivant:

As at Caen in Normandy, now in the Reichswald forest, it fell to British troops [et canadiennes] to fight the second and last of the great battles of the "hinge", and to open the way ${ }^{90}$.

Plus loin, il s'explique en ces termes:

By the fortunes of war the Canadians and the British on the left had held the hinge, as they had done in Normandy, taking the full weight of the enemy defensive battle, and opening the way for the brilliant drives of the American armour on the right. By its battle of the Rhineland the Canadian 1st Army had cleared the road to Cologne for the U.S. 1st Army ${ }^{91}$.

87 Eisenhower, op. cit., 210.

88 Thompson, op. cit., 230.

89 Schulman, op. cit., 320.

90 Thompson, op. cit., 4.

91 Ibid., 230. 
Voici, de son côté, comment le maréchal Montgomery a décrit la résistance ennemie contre les Canadiens durant cette bataille:

The enemy parachute troops fought with a fanaticism unexcelled at any time in the war, and it is interesting to note that the Germans had available against the Canadian Army some 700 mortars and over 1,000 guns of all types; the volume of fire from enemy troops was the heaviest which had been met so far ... in the campaign ${ }^{92}$.

\section{TÉMOIGNAGE D'UN COMBATTANT}

Le 10 février, un officier du Régiment faisait part de ses impressions sur la récente bataille, dans les termes suivants:

Depuis l'offensive canadienne du 8 février, exactement six mois après l'offensive sur Falaise, nous avons revu les mêmes scènes qu'en Normandie: ruines, cadavres, animaux morts, prisonniers, etc. Encore hier soir, deux... déserteurs de la Wehrmacht échouaient chez nous... C'était deux parachutistes, c'est-à-dire appartenant à ce qui fut un jour l'élite de la jeunesse hitlérienne. Tandis que le sergent-major du Régiment en fouillait un, je fouillais l'autre. Le premier avait un regard cynique et moqueur; l'autre, plus timide, tremblait de frayeur. Le commandant a gardé la Croix de fer que le premier portait; il avait gagné cette décoration en Russie... La race supérieure, quelle blague !... Pour les Boches, c'est plus que jamais leur "Götterdämmerung" ${ }^{93}$.

L'opération "Veritable", que le général Bedell Smith, chef d'état-major d'Eisenhower, a considéré comme "one of the most bitterly fought actions of the entire war in the West ${ }^{94}$ ", était maintenant terminée.

92 Montgomery, op. cit., 196.

93 Archives de l'auteur [Labbeck], 10 mars 1945.

${ }^{94}$ General Walter Bedell Smith, Eisenhower's Six Great Decisions (New-York, Longmans, Green and Co., 1956), 135. 


\section{RETOUR EN HOLLANDE}

Dès le 12 mars 1945, le Régiment recevait l'ordre "pour une raison ignorée d'un grand nombre ${ }^{95}$ ", de retourner en Hollande, sur un front beaucoup plus calme, sinon complètement inactif, en attendant de revenir en Allemagne, cette fois pour franchir le Rhin et pour participer, de nouveau avec les valeureuses troupes polonaises, à l'écrasement définitif de la Wehrmacht, aux portes de Wilhemshaven sur la Baltique.

\section{(à suivre)}

\section{JACQUES GOUIN,}

ex-lieutenant d'artillerie, diplômé en sciences politiques (Ottawa), correspondant canadien $\grave{a}$ la Revue d'Histoire de la $2^{\mathrm{e}}$ guerre mondiale (France), chef adjoint du Bureau des traductions et rédacteur de l'édition française du Journal de l'Armée canadienne, ministère de la Défense nationale, Ottawa.

${ }^{95}$ Lieut.-col. J.-H.-R. Gagnon et lieuts P.-L. Côté, J.-R. Gouin et P.-M. Pelletier, The History of the 4 th Canadian Medium Regt RCA, (Hollande, 1945), 13. 